\title{
MANAGING PLACENTA ACCRETA SPECTRUM IN LOW RESOURCE SETTING USING A NOVEL DISSECTION-FREE AORTA CLAMP: OPERATIVE TECHNIQUE
}

\author{
Vakkanal Paily ${ }^{1}$, Afshana Sidhik ${ }^{1}$, Raji Raj ${ }^{1}$, Ajithakumari Sudhamma ${ }^{1}$, Joshy Joseph ${ }^{1}$, \\ M.G Usha ${ }^{1}$, Raymond George ${ }^{1}$, Soumya Ramakrishnan ${ }^{1}$, Sara Cheriyan ${ }^{1}$, Teena John ${ }^{1}$, and \\ Manu Pradeep ${ }^{1}$ \\ ${ }^{1}$ Rajagiri Hospital
}

November 8, 2021

\begin{abstract}
Objective: Surgical management of Placenta accreta spectrum (PAS) is associated with profuse bleeding and increased risk of operative injury to the adherent pelvic structures. We propose the use of a novel aorta clamp that can occlude the abdominal aorta, without retro-peritoneal dissection, thereby making it easy for the obstetrician to use it. limiting the incident blood loss.

Methods: This is a retrospective chart review of 33 women, with varying grades of histopathology confirmed PAS, who were managed as an elective or emergency procedure in a tertiary center in India. In all cases, the novel Paily Aorta Clamp (PAC) was applied just above the bifurcation of the abdominal aorta.

Results: Twenty-nine women with advanced grades of PAS, underwent sub-total hysterectomies while four women with low grade (focal) PAS underwent a conservative procedure. The procedures were associated with median estimated intra-operative blood loss of $1000 \pm 1500 \mathrm{ml}$ with only $51.5 \%(\mathrm{n}=17)$ requiring any blood transfusions. PAC was applied for a median $55 \pm$ 20 minutes and was not associated with any peri-operative aortic wall injury or distal thromboembolic phenomenon.
\end{abstract}

Conclusion: Aortic clamping is feasible without retroperitoneal dissection using the PAC, which can be used to limit operative blood loss and surgical morbidity in PAS disorders.

\section{INTRODUCTION}

Rising cesarean section rate is a global concern, affecting developing and developed countries [1]. Along with high cesarean section rate comes a high incidence of Placenta Accreta Spectrum (PAS) [2]. PAS management is a nightmare to obstetricians across the world, because of the severe bleeding that ensues while attempting to separate the placenta from the surrounding structures like the bladder [3].

The developed countries manage PAS disorders, by organizing multi-disciplinary teams consisting of vascular/onco-surgeons, hematologists and urologists in addition to senior obstetricians and anesthesiologists [3]. Blood bank and critical care infrastructure enable them to transfuse massive quantities of blood as necessitated and can even deploy cell salvage systems to reuse the lost blood[3]. To reduce bleeding, often intravascular balloon occlusion is used at the lower end of abdominal aorta or the common iliac arteries [3], [4]. Developing countries also face the problem of PAS, but the resources described above are rarely available - leading to severe morbidity and even mortality.

We describe our experience with a novel aorta clamp - Paily Aorta Clamp (PAC) developed by us, to manage placenta accreta spectrum in a center with limited resources.

MATERIAL AND METHODS 
Between April 2015 and May 2021, Rajagiri Hospital - Kerala, India served as a referral center for Placenta Accreta Spectrum. All mothers managed with Paily Aorta Clamp (PAC), and a histopathological diagnosis of PAS [5], were included in the study. Institutional Ethics Committee - Rajagiri Hospital, Aluva, Kerala, India approved this study on $18^{\text {th }}$ August 2021 - vide ref. RAJH2021015.

Conceptualised by Dr. Paily in 2006, PAC (Babu Surgicals, Palakkad, Kerala, India) is a simple straight instrument, which looks like an Allis forceps (Figure 1) but with blunt tips which overlap and act as a guard to prevent the vessel slipping out of the clamp (Figure 2). The blades feature a smooth inner surface and even on maximum closure, there is a gap of about $2 \mathrm{~mm}$ between the blades to prevent crush injury to the vessel wall and vaso-vasorum of the aorta (Figure 2, Figures S1 \& S2).

Elective procedures were scheduled before 36 weeks of gestation, after being counselled about the risks of surgery. They were evaluated by USG or MRI[6]. Their pre-operative risks (like anemia) were optimised and adequate blood products arranged in anticipation of massive transfusion -4 units each of packed Red Blood Cells (pRBC), Fresh Frozen Plasma (FFP), Cryoprecipitate (CPP) and single donor platelets (SDP). A written informed consent for the prophylactic or emergent use of PAC, was obtained prior to all procedures.

Preoperatively, the women are prepared in supine position with left lateral tilt, placed under spinal/general + epidural anesthesia with the bladder catheterised. Intermittent pneumatic compression device for Deep Vein Thrombosis (DVT) prophylaxis is initiated. In women with suspected bladder involvement, the ureters are also catheterised until the end of the surgery. Inj. Tranexamic Acid 1gm in 100 $\mathrm{ml}$ normal saline was occasionally administered to select patients with suspected high grade of placental invasion.

As an elective procedure for high grade PAS, we prefer a vertical midline incision on the abdominal wall with extension above the umbilicus as required. Uterus is incised above the level of the placenta and the fetus is delivered. The umbilical cord is clamped and cut. Bleeding from the uterine wound is controlled with Green Armytage forceps, the uterus is exteriorised and pushed caudally.

To apply the PAC, after pushing the bowels laterally, the lower end of abdominal aorta is identified above the bifurcation by palpation and gently lifted up using a Babcock forceps. The PAC is applied making sure that the entire wall of aorta is between the blades, with the guard at the tip going beyond, securing the vessel from slipping out. There is no need for any peritoneal dissection or vessel skeletonization; thus, avoiding the risk of injury to inferior vena cava or the posterior lumbar vessels (Video 1). Only the minimum occlusive clamping force is applied, , stopping when the pulsations cease in the common iliac arteries.

Electively, a sub-total hysterectomy is then performed by sequential clamping and cutting of the pedicles. If it is anterior percreta placenta, with meticulous care, the bridging vessels between bladder and placenta are coagulated and cut. The membranous coagulum on the surface of the placenta is ideally left undisturbed, as it tends to bleed profusely if disrupted. The lower level of the placenta is then identified by compressing the anterior and posterior walls of the lower segment, between the fingers. The cervix is transversely clamped and transected at below this (Figure 3) with the cervical stump being closed with box stitches. Care is taken to ensure that no placental tissue is left behind. Further attempts at bladder separation from the cervix and vagina below this level is avoided, as hemostasis is arduous in this area. However, if the woman is in labor and the cervix has already dilated, then a total hysterectomy maybe warranted.

In cases where PAS is encountered unexpectedly during emergency/elective cesarean delivery with a transverse, suprapubic abdominal incision, access to the aorta can be difficult. Hence, we clamp the bilateral common iliac arteries using the same technique with the PAC, at the pelvic brim. If there is only focal accreta, the uterus can be conserved after excision of the morbidly adherent part of the placenta.

The PAC is applied for as short a duration as possible and released after the hysterectomy/placental removal. However, in difficult pelvic dissections, the clamp was left occluding the vessel even up to 90 minutes. Application of the clamp and its removal is announced loud and the duration of clamp application is noted. The operating obstetrician confirms return of circulation, by palpating the pulsations in the external iliac arteries. 
If major bleeding persisted post-clamp release, the clamp can be reapplied in the same location (Video S2), to facilitate rapid control of bleeding sites. Post-clamp release, as an additional hemostatic measure, bilateral internal iliac artery ligation can be performed after hysterectomies if the bladder base still had bleeding [7]. In conservative procedures: step-wise devascularization, uterine compressive sutures or Bakri balloon application can be performed if hemorrhage ensues post-clamp release.

Intra-operative blood loss (EBL) was estimated by i) amount collected in suction containers ii) the increase in weight of blood-soaked surgical pads $(1 \mathrm{gm}=1 \mathrm{ml})$ and iii) visual estimation of blood spilled over the floor, if any. Since our team used the same suction and pads to clear the amniotic fluid, this EBL may be overestimated. All post-operative specimens were sent for histopathological examination.

Post-operatively, the bladder is left catheterised for 24 - 48 hours (depending on the degree of bladder base dissection). DVT prophylaxis using Low Molecular Weight Heparin and compression stockings are provided until ambulant. Intensive care (ICU) is provided for women who received $>4$ units of pRBC) or developed other complications. The women were routinely discharged at post-op day 7 and followed-up in person, after 6 weeks. In two women (P31 \& P32), we performed an MRI Aortogram during this visit to examine post-clamping aortic wall integrity, as they had endured a prolonged clamp application ( $>100$ minutes).

The required maternal baseline characteristics, peri-operative outcomes and histopathological reports were retrospectively abstracted from labor registries/operative notes and used to classify the final diagnosis into various grades of PAS as recommended by FIGO[5]. Distribution of nominal variables will be reported with frequency (percentage) and Pearson's $\chi^{2}$ tests, whereas continuous variables will be reported with mean \pm S.D and one-way ANOVA (or median \pm IQR and Kruskal Wallis as specified), when the cases were classified according to their final PAS grades. A p $<0.05$ was determined to have statistical significance. All statistical analysis was performed on SPSS version 25 (IBM, Armonk, NY, USA).

\section{RESULTS}

The detailed maternal pre-operative demographics and intra-operative and post-operative surgical outcomes have been summarised in Tables S1, S2 and S3 respectively, stratified for histopathology-confirmed PAS grades. Twenty-nine women with advanced grades of PAS, underwent sub-total hysterectomies while four women with focal accreta $(\mathrm{n}=4)$, underwent a conservative procedure.

In general (Table 1), the procedures (including ureteric catheterization) lasted a median of $182 \pm 45$ [Range $=120-460$ ] minutes, with PAC applied for a median duration of $55 \pm 20$ minutes [Range $=30-165$ ] minutes. The median measured blood loss was $1000 \pm 1500$ [Range: $300-5000$ ] ml with only $51.5 \%(\mathrm{n}=17)$ of cases requiring transfusion, and only $21.2 \%(\mathrm{n}=7)$ required a massive transfusion of more than 4 units of pRBC. Median transfusion components required were $1 \pm 3$ units of $\mathrm{pRBC}, 0 \pm 2$ units of fresh frozen plasma (FFP). In those who did not receive a transfusion, the median drop in post-operative hemoglobin was only $0.7 \pm 1.7 \mathrm{gm} / \mathrm{dl}$.

A woman (P11) had excess blood loss $(3000 \mathrm{ml})$ as she underwent an emergency hysterectomy while on Low Molecular Weight Heparin for an antenatal onset pulmonary embolism. Another woman (P26) endured excessive blood loss (5000ml) and extended clamp time (165 minutes); she was admitted with antepartum hemorrhage $(2000 \mathrm{ml})$ and underwent emergency hysterectomy at odd hours without adequate preparation or experienced manpower. Another woman (P12) had excess blood loss (4000ml) due to right internal iliac vein injury sustained while attempting internal iliac artery ligation as a prophylactic measure and was repaired by a cardiothoracic-vascular surgeon. A woman (P31) had extensive placental invasion of the bladder and left parametrium which necessitated an arduous pelvic dissection with a prolonged operative time (460 minutes) and total clamp time (155 minutes) with the urologist repairing the bladder laceration.

A woman (P5) suffered an inadvertent ureteric injury during ureteric catheterization and required repair by an urologist. Six women suffered bladder injuries - three of them were repaired by urologists and the rest by senior obstetrician. All injuries to urinary tract healed uneventfully at an in-person follow up at a median $6.5 \pm 2$ weeks. Two women developed pelvic hematomas in the post-operative period, which were managed 
conservatively and resolved uneventfully. Other complications are detailed in Table. S2 and S3.

None of the women developed any signs of aortic wall rupture, reperfusion injury, lower limb thromboembolic phenomenon or abdominal-pelvic organ ischemia in the immediate post-operative period ( $<3$ days) and up to a $6^{\text {th }}$ week follow-up visit, with three women lost to follow-up. The 2 women (P31 \& P32) who underwent a MRI Aortogram, did not show any signs of aortic wall pseudo-aneurysm or dissection (Figure S3).

\section{DISCUSSION}

Using the PAC, 33 women of varying grades of PAS had positive surgical outcomes with no clamp related adverse events.

Obstetricians around the globe seek innovative means to reduce: i) the catastrophic amounts of blood loss (median $=2000-3000 \mathrm{ml})$, a high probability of urological injuries (7-48\%) and iii) massive transfusion requirements $(\mathrm{pRBC}=3.4$ to $5.4 \mathrm{~L})$ encountered during non-conservative surgery [3].

The proximal occlusion of blood supply may resolve the emergent threats of placenta accreta spectrum. Hardly a novel idea, cardiovascular surgeons have cross clamped the abdominal aorta for aneurysmal repair for up to 150 minutes, and reports a low risk of immediate adverse events [8]. This technique, when adopted into PAS management and reported as small, single center trials by Chou et. al 2021, Joshi et. al 2021 and Sucu et.al 2020 (Common iliac artery), reveals promising results with radically reduced average estimated blood loss $(595 \mathrm{ml}-2296 \mathrm{ml})$ with only 6.5 to $25 \%$ of urological injuries reported and an average 2 to 6 units of pRBC transfusion requirement. Reassuringly, none reported any distal thromboembolic phenomena [9]-[11]. Thus, aortic cross-clamping reveals similar effectiveness, feasibility and safety profile at least on par with or even superior to aortic balloon occlusion, pending comparative trials [12].

However, we believe cross clamping still relies on the expertise of a vascular surgeon or obstetrician capable of retroperitoneal dissection, risking vulnerable adjacent veins. It may not be apt in an emergent situation, where the obstetrician may not possess the time nor the skill, in all resource settings.

Over-the-peritoneum, dissection-free clamping using PAC, is similarly effective, arguably safer and easier to apply for an obstetrician in a low resource setting. The atraumatic, secure design of PAC ensures a relatively blood less field for complex pelvic dissection, (especially bladder base dissection) in high grade PAS. We acknowledge the salience of a multi-disciplinary team to manage emergent peri-operative complications.

\section{CONCLUSION}

Over-the-peritoneal clamping of the abdominal aorta is an effective, safe and feasible technique that we propose in low-resource settings, pending further larger, prospective trials. This small case series demonstrates promising peri-operative outcomes in the surgical management of PAS disorders, while minimizing the dependence of obstetricians on interventional radiologists, and vascular surgeons.

Acknowledgements: This study was possible with the whole hearted support of the women under our care. We received invaluable support from our colleagues from the departments of Anesthesiology, Radiology, Urology, Cardiothoracic-Vascular Surgery and Pathology along with Junior Residents in our department. We are grateful to the Institutional Scientific and Ethics committees as well as the administrative, nursing and support staff of Rajagiri Hospital, Kerala, India. We also wish to mark our gratitude to Ms. Babitha S. Meleth for aiding in data collection/secretarial assistance.

Disclosure of Interests: First author, Dr. V.P Paily has applied for a patent for the "Paily Common Iliac/Aorta Clamp" (No.292/CHE/2012) in India. Completed disclosure of interest forms are available to view online as supporting information.

Author Contributions: VP Paily designed the Paily Aorta Clamp and its technique. VP Paily, Afshana Sidhik and Manu Pradeep designed and collected data for the study. The operative team consisted of VP Paily, Afshana Sidhik, Raji Raj G., Ajithakumari Sudhamma, Joshy Joseph, M. G. Usha, Raymond George, Soumya Ramakrishnan, Sara Cheriyan, Teena Eliz John who were principal obstetricians or assistants who 
undertook the procedures. Manu Pradeep performed data analysis. VP Paily and Manu Pradeep wrote the first draft of this article with all other authors contributing critical revisions.

Details of Ethics Approval: Institutional Ethics Committee - Rajagiri Hospital, Aluva, Kerala, India approved this study on $18^{\text {th }}$ August 2021 - vide ref. RAJH2021015.

Funding: This study was conducted as part of the author's employment in Rajagiri Hospital, Aluva, Kerala, India and did not receive any specific funding from any external source.

\section{REFERENCE}

[1] J. E et al., "FIGO consensus guidelines on placenta accreta spectrum disorders: Introduction," International Journal of Gynecology and Obstetrics, vol. 140, no. 3, pp. 261-264, Mar. 2018, doi: 10.1002/ijgo.12406.

[2] E. Jauniaux et al., "FIGO consensus guidelines on placenta accreta spectrum disorders: Epidemiology," International Journal of Gynecology and Obstetrics, vol. 140, no. 3, pp. 265-273, Mar. 2018, doi: 10.1002/ijgo.12407.

[3] L. Allen et al., "FIGO consensus guidelines on placenta accreta spectrum disorders: Nonconservative surgical management," International Journal of Gynecology and Obstetrics, vol. 140, no. 3, pp. 281-290, Mar. 2018, doi: 10.1002/ijgo.12409.

[4] E. R. M. Jauniaux et al., "Placenta Praevia and Placenta Accreta: Diagnosis and Management: Greentop Guideline No. 27a," BJOG: An International Journal of Obstetrics and Gynaecology, vol. 126, no. 1, pp. e1-e48, 2019, doi: 10.1111/1471-0528.15306.

[5] E. Jauniaux et al., "FIGO classification for the clinical diagnosis of placenta accreta spectrum disorders," International Journal of Gynecology and Obstetrics, vol. 146, no. 1, pp. 20-24, Jul. 2019, doi: 10.1002/ijgo.12761.

[6] E. Jauniaux, A. Bhide, A. Kennedy, P. Woodward, C. Hubinont, and S. Collins, "FIGO consensus guidelines on placenta accreta spectrum disorders: Prenatal diagnosis and screening," International Journal of Gynecology $\& 3$ Obstetrics, vol. 140, no. 3, pp. 274-280, Mar. 2018, doi: 10.1002/IJGO.12408.

[7] V. P. Paily, "Initial Interventions to Combat Hemorrhage during Cesarean Section and Internal Iliac Artery Ligation," in A Comprehensive Textbook of Postpartum Hemorrhage, 2nd ed., A. Sabaratnam, M. Karoshi, L. G. Keith, A. B. Lalonde, and C. B-Lynch, Eds. Sapiens Publishing, 2012, pp. 455-457.

[8] N. J et al., "Safe time limits of aortic cross-clamping and cardiopulmonary bypass in adult cardiac surgery," Perfusion, vol. 24, no. 5, pp. 297-305, 2009, doi: 10.1177/0267659109354656.

[9] S. Sucu, H. Ç. Ozcan, M. Misirlioglu, O. Guralp, and B. Kaya, "Temporary Clamping of Bilateral Common Iliac Artery During Cesarean Hysterectomy for the Management of Placenta Percreta," European Journal of Obstetrics and Gynecology and Reproductive Biology, vol. 250, pp. 188-194, Jul. 2020, doi: 10.1016/J.EJOGRB.2020.04.021.

[10] V. M. Joshi, S. R. Otiv, Y. B. Sovani, and P. K. Kulat, "Aortic clamping for limiting blood loss at cesarean hysterectomy for placenta percreta: A case series," International Journal of Gynecology \& Obstetrics, 2021, doi: 10.1002/IJGO.13746.

[11] M. Chou et al., "Vascular control by infrarenal aortic cross-clamping in placenta accreta spectrum disorders: description of technique," BJOG: An International Journal of Obstetrics 8 Gynaecology, vol. 128, no. 6, pp. 1030-1034, May 2021, doi: 10.1111/1471-0528.16605.

[12] L. Chen et al., "Clinical evaluation of prophylactic abdominal aortic balloon occlusion in patients with placenta accreta: A systematic review and meta-analysis," BMC Pregnancy and Childbirth, vol. 19, no. 1, pp. 1-8, Jan. 2019, doi: 10.1186/s12884-019-2175-0. 


\section{Figures and Table Legends}

Figure 1. Paily Aorta Clamp (PAC) - Open position

Figure 2. Paily Aorta Clamp (PAC) - Distal end closed with 2mm gap between blades.

Figure 3. An operative specimen of Placenta Percreta dissected away from bladder base; Green arrow indicates the obstetrician's finger in the transected cervix.

Table 1. Unstratified data set of all women with PAS who received the Paily Aorta Clamp

\section{Supporting Information}

Video S1. Intraoperative video demonstrating the application of Paily Aorta Clamp (PAC) on the abdominal aorta.

Video S2. Rapid resolution of intraoperative hemorrhage after PAC re-application.

Figure S1. Paily Aorta Clamp (PAC) - Distal end with vessel slip guard

Figure S2. Paily Aorta Clamp (PAC) - Distal end with vessel slip guard - Other perspective

Figure S3. Representative MRI Aortogram where the clamp was applied for a total of 155 minutes $(80+$ 75 minutes). There is no evidence of local injury or dilation.

Table S1: Maternal clinical demographics - categorised by histopathological grades of Placenta Accreta Spectrum.

Table S2: Operative parameters and outcomes - categorised by histopathological grades of Placenta Accreta Spectrum.

Table S3. Post-operative and follow-up characteristics - categorised by histopathological grades of Placenta Accreta Spectrum.

\section{Hosted file}

Print Figures.docx available at https://authorea.com/users/437504/articles/539092-managingplacenta-accreta-spectrum-in-low-resource-setting-using-a-novel-dissection-free-aortaclamp-operative-technique

\section{Hosted file}

Print Table.docx available at https://authorea.com/users/437504/articles/539092-managingplacenta-accreta-spectrum-in-low-resource-setting-using-a-novel-dissection-free-aortaclamp-operative-technique 